\title{
Variabilidade espacial de atributos químicos em diferentes profundidades em um Latossolo em sistema de plantio direto
}

\author{
Luiz Antônio Zanão Júnior ${ }^{1}$ Regina Maria Quintão Lana² Maristela Pereira Carvalho- Zanão ${ }^{3}$ \\ Ednaldo Carvalho Guimarães ${ }^{4}$
}

\begin{abstract}
RESUMO
O estudo da variabilidade espacial dos atributos químicos no solo é uma etapa importante na agricultura de precisão. Objetivou-se com este trabalho avaliar a variabilidade espacial em duas profundidades $(0-10$ e $10-20 \mathrm{~cm})$ do pH em água, saturação por bases e teores de matéria orgânica e micronutrientes (B, Cu, Fe, Mn e Zn) em um Latossolo Vermelho distrófico, textura média, cultivado há oito anos em sistema de plantio direto, em Uberlândia - MG. A coleta das amostras do solo foi realizada em intervalos regulares de $50 \mathrm{~m}$, em malha regular de 121 pontos. Os dados foram avaliados por estatística descritiva e geoestatística, com base no ajuste de semivariogramas. Maiores teores de matéria orgânica, Mn e Zn foram verificados nos primeiros $10 \mathrm{~cm}$ do solo. A maior variabilidade foi encontrada para B e a menor para pH em água. Além da variabilidade vertical, há também variabilidade horizontal entre as profundidades. A dependência espacial varia conforme o atributo químico avaliado e a profundidade de coleta. Os valores de alcance foram maiores na profundidade de $0-10 \mathrm{~cm}$.
\end{abstract}

Palavras-chaves: Geoestatística, fertilidade do solo, semivariograma, amostragem

\section{ABSTRACT}

\section{Spatial variability of chemical attributes at different depths in an Oxisol under no-till system}

The study of spatial variability of soil chemical attributes is an important step in precision agriculture. The objective of this study was to evaluate the spatial variability at two depths (0-10 and 10-20 cm) of the pH in water, base saturation, and levels of organic matter and micronutrients (B, Cu, Fe, Mn and $\mathrm{Zn}$ ) in an Oxisol, medium texture, under no-tillage system for eight years. Soil sample collection was performed at regular intervals of $50 \mathrm{~m}$ in a regular grid of 121 points. Data were analyzed by descriptive statistics and geostatistics based on the adjustment of semivariograms. Higher levels of organic matter, $\mathrm{Zn}$ and $\mathrm{Mn}$ were recorded in the first $10 \mathrm{~cm}$ of soil. The greatest variability was found for $\mathrm{B}$ and the lowest for $\mathrm{pH}$ in water. Most of the data fit the model of Gaussian semivariogram. The values of range were different as a function of the sampling depth and greater at the depth 0 $10 \mathrm{~cm}$. If a geostatistical sampling scheme is used, the number of samples to be collected will be different for the two depths and among the evaluated attributes.

Key words: Geoestatistic, soil fertility, semivariogram, sampling

\footnotetext{
Recebido para publicação em abril de 2009 e aprovado em março de 2010

'Engenheiro-Agrônomo, Mestre. Instituto Agronômico do Paraná (IAPAR), Pólo Regional de Pesquisa de Ponta Grossa, Caixa Postal 129, 84001-970, Ponta Grossa, Paraná, Brasil. lzanao@iapar.br.

${ }^{2}$ Engenheira-Agrônoma, Doutora. Instituto de Ciências Agrárias, Universidade Federal de Uberlândia (UFU), Caixa Postal 593, 38400-902, Uberlândia, Minas Gerais, Brasil. rmqlana@iciag.ufu.br.

${ }^{3}$ Engenheira-Agrônoma, Mestre. Rodovia do Café, Km 496, Caixa Postal 129, 84001-970, Ponta Grossa, Paraná, Brasil. maristelacarvalho@gmail.com.

${ }^{4}$ Engenheiro Agrícola, Doutor. Faculdade de Matemática, Universidade Federal de Uberlândia (UFU). Caixa Postal 593, 38408-100, Uberlândia, Minas Gerais, Brasil. ecg@ufu.br.
} 


\section{INTRODUÇÃO}

O solo, por ser um corpo tridimensional, formado pela ação de vários processos e sob diversos manejos, apresenta grande variação de suas características nos sentidos vertical e horizontal. Esta variabilidade espacial dos atributos do solo pode ser medida e registrada (Vieira et al., 1983). Conhecer essa variabilidade é importante passo para que se possa empregar um manejo mais adequado, considerando estratégias de amostragem, aplicação de fertilizantes, além de planejamento de delineamento de pesquisa em campo (Bhatti et al., 1991).

Os princípios básicos da experimentação estabelecida por meio do método estatístico clássico consideram que a variabilidade do solo ocorre de forma inteiramente aleatória, admitindo-se que os erros associados aos valores de seus atributos apresentam distribuição de frequência normal (Santos \& Vasconcelos, 1987). Amostragens ao acaso também podem ser feitas, obtendo-se estimativas de valores médios e de variância. Nesses casos, a variabilidade é descrita pelo coeficiente de variação (Reichardt et al., 1986). Entretanto, vários estudos mostraram que os atributos do solo apresentam intensa dependência espacial, verificada por análise geoestatística (Santos \& Vasconcelos, 1987; Souza et al., 1997; Corá et al., 2004; Bekele \& Hudnall, 2006; Motomiya et al., 2006; Souza et al., 2006; Cavalcante et at., 2007; Zanão Júnior et al., 2007; Campos et al., 2008).

A geoestatística pode ser empregada no caso de amostragens com distâncias conhecidas, determinandose a variabilidade espacial do atributo por semivariogramas que definem o grau de dependência no espaço de cada amostragem realizada (Reichardt et al., 1986). Assim, é possível determinar espaçamentos entre pontos de amostragem para os quais as médias de um atributo do solo sejam realmente independentes entre si.

No sistema plantio direto, a aplicação superficial uniforme do calcário, os diferentes resíduos das culturas usadas em sucessão ou rotação ao longo dos anos deixados na superfície, a ausência de revolvimento do solo por práticas como aração e gradagem e as frequentes adubações em superfície, a lanço ou nas linhas de cultivo de culturas de variados espaçamentos, alteram a variabilidade dos teores de macro e micronutrientes no solo e de matéria orgânica, além do $\mathrm{pH}$ em relação ao sistema convencional, tanto no sentido vertical quanto no horizontal (Souza, 1992; Salet et al., 1996; Schlindwein \& Anghinoni, 2000; Trevisan et al., 2008). Assim, essa variabilidade espacial dos atributos do solo no sistema plantio direto resulta na necessidade da definição de novos procedimentos de amostragem para contemplar tais alterações.
Uma das maneiras mais utilizadas para representar a variabilidade espacial é por meio de mapas de isolinhas (Burgess \& Webster, 1980). Para tanto, é necessário conhecer as coordenadas geográficas dos locais onde foram coletadas as amostras e o grau de dependência espacial entre as amostras, que pode ser avaliado pelos semivariogramas. Assim, os estudos da variabilidade espacial dos valores dos atributos do solo de uma propriedade agrícola permitirão a obtenção de mapas de fertilidade de solos e o estabelecimento de estratégias de aplicação de fertilizantes (Burrough et al., 1996).

Para uma amostragem representativa da fertilidade de uma área é necessário o conhecimento da variabilidade espacial do atributo a ser trabalhado. Assim, objetivou-se com este trabalho avaliar, em duas profundidades, a variabilidade espacial do pH em água, saturação por bases e teores de matéria orgânica e micronutrientes $(\mathrm{B}, \mathrm{Cu}$, Fe, Mn e Zn) em um Latossolo Vermelho distrófico, textura média, cultivado há oito anos no sistema plantio direto.

\section{MATERIAL E MÉTODOS}

A área experimental localiza-se entre as coordenadas geográficas $19^{\circ} 06^{\prime}$ latitude sul e $48^{\circ} 51^{\prime}$ longitude oeste e altitude média de $650 \mathrm{~m}$, em área particular, na Fazenda São Luiz de Jaborandi, no município de. Uberlândia - MG. O solo é classificado como Latossolo Vermelho distrófico típico, textura média ( $250 \mathrm{~g} \mathrm{~kg}^{-1}$ de argila). O relevo é predominantemente suave ondulado, com declividade média de 3 a 6\%. O clima da região, segundo a classificação de Köppen, é Cwa, com estação seca bem definida de maio a setembro e pluviosidade média anual de $1.500 \mathrm{~mm}$.

O sítio de amostragem está sob cultivo de culturas anuais há mais de 20 anos e há oito anos sob sistema de plantio direto. Esses dados foram coletados no período de maio a junho de 2003. A rotação de culturas durante os cinco primeiros anos nesse sistema foi milho na resteva de soja e 30\% da área com milho no verão a partir da safra 1995/96. Após a colheita do milho safrinha foram utilizados milheto, nabo forrageiro ou aveia branca como cultura de cobertura de solo, cujas sementes sempre foram semeadas a lanço.

Na implantação do sistema plantio direto no ano de 1995 foram aplicados e incorporados gesso agrícola (1,5t ha $^{-1}$ ) e calcário (visando elevar a saturação por bases para 65\%). Nas safras de 1998/99 e 2001/02 houve nova aplicação de calcário, também visando elevar a saturação por bases para 65\%. No período de 1996 a 2000, a adubação de semeadura para a cultura da safra de verão (soja ou milho) foi realizada no sulco de semeadura, na base de 140 
kg ha-1 de N, $100 \mathrm{~kg} \mathrm{ha}^{-1}$ de $\mathrm{P}_{2} \mathrm{O}_{5}, 90 \mathrm{~kg} \mathrm{ha}^{-1}$ de $\mathrm{K}_{2} \mathrm{O}$ e 2 kg ha $^{-1}$ de $\mathrm{Zn}$. Quando a cultura de verão era a soja, a adubação nitrogenada era suprimida. As culturas de cobertura de solo, milheto, nabo forrageiro ou aveia branca não receberam adubação. Na safra 2000/2001, a cultura de verão foi a soja, com aplicação de $400 \mathrm{~kg} \mathrm{ha}^{-1}$ do formulado 02-20-18 $+1 \mathrm{~kg} \mathrm{ha}^{-1}$ de B no sulco de semeadura, Co (2 g ha-1) e Mo (20 $\mathrm{g} \mathrm{ha}^{-1}$ ) no tratamento de sementes e, Mn via foliar (300 $\mathrm{g} \mathrm{ha}^{-1}$ ); na safra 2001/2002, a cultura de verão foi o milho, com aplicação de $500 \mathrm{~kg} \mathrm{ha}^{-1}$ do formulado 08-28-18 + 6 kg ha ${ }^{-1}$ de $\mathrm{Zn}$ na semeadura e $120 \mathrm{~kg} \mathrm{ha}^{-1}$ de uréia em cobertura (lanço). Na safra 2002/2003, a cultura de verão foi o algodão, com aplicação de $650 \mathrm{~kg} \mathrm{ha}^{-1}$ do formulado 0828-16 + 1 kg ha-1 de Zn no sulco de semeadura.

Em 2003, após a colheita do algodão, uma parcela de 500 x 500 m foi dividida numa malha com espaçamento regular de $50 \mathrm{~m}$ entre os pontos, resultando um quadrado de 11 linhas e 11 colunas, totalizando 121 pontos amostrados nos cruzamentos de malha. Todos os pontos amostrados foram georreferenciados, sendo retirada uma amostra simples por ponto. As amostras foram retiradas com pá de corte transversalmente aos sulcos de semeadura e no espaço compreendido entre os pontos médios entre os sulcos e estratificadas em duas profundidades, 0-10 e $10-20 \mathrm{~cm}$.

As amostras de solo após serem secas ao ar e peneiradas em malha de $2 \mathrm{~mm}$ de abertura foram analisadas quimicamente, determinando-se $\mathrm{pH}$ em água na relação 1:2,5 (solo-água), matéria orgânica, boro (água quente), ferro, cobre, manganês e zinco disponíveis (Mehlich-1), conforme procedimentos apresentados por Silva (1999). Os resultados encontrados foram primeiramente submetidos à análise estatística descritiva, obtendo-se média, mediana, valor máximo, valor mínimo, coeficiente de variação, coeficiente de assimetria e coeficiente de curtose. O teste t Student, também a 5\% de probabilidade, foi aplicado para comparação das médias dos atributos entre as profundidades.

A dependência espacial dos atributos estudados foi avaliada por meio da geoestatística (Vieira et al., 1983), por meio do cálculo da semivariância e do ajuste dos dados ao semivariograma experimental. Os semivariogramas são representações gráficas entre a semivariância $\gamma(\mathrm{h})$ representada na coordenada y, em função de determinada distância h, representada na coordenada x. A semivariância pode ser estimada pela seguinte equação:

$$
\gamma(\mathrm{h})=\frac{1}{2 \mathrm{~N}(\mathrm{~h})} \sum_{\mathrm{i}=1}^{\mathrm{N}(\mathrm{h})}\left[\mathrm{Z}\left(\mathrm{x}_{\mathrm{i}}\right)-\mathrm{Z}\left(\mathrm{x}_{\mathrm{i}}+\mathrm{h}\right)\right]^{2}
$$

em que $N$ (h) representa o número de pares de valores medidos, Z(xi) e Z(xi $+\mathrm{h}$ ), separados por um vetor $(\mathrm{h})$. $\mathrm{O}$ gráfico de $\gamma(\mathrm{h})$ versus os valores correspondentes de h é denominado semivariograma.

Os semivariogramas foram ajustados com o software $\mathrm{Gs}^{+}$(Gamma Design Software, 2000). A seleção dos modelos foi realizada com base no melhor coeficiente de determinação $\left(\mathrm{R}^{2}\right)$ e na menor soma de quadrados do resíduo (SQR), sendo testados os modelos esférico, exponencial, gaussiano, linear, linear sem patamar e efeito pepita puro. Nos semivariogramas ajustados foram definidos os seguintes parâmetros: efeito pepita $\left(\mathrm{C}_{0}\right)$; patamar $\left(\mathrm{C}_{0}+\mathrm{C}_{1}\right)$; e alcance da dependência espacial (a). A razão entre o efeito pepita e o patamar $\mathrm{C}_{0} /\left(\mathrm{C}_{0}+\mathrm{C}_{1}\right)$ permite a classificação e a comparação entre atributos do solo. Para analisar o grau de dependência espacial dos atributos em estudo, utilizou-se a classificação proposta por Cambardella et al. (1994), em que os semivariogramas com dependência espacial forte possuem razão menor ou igual a $25 \%$, os com dependência espacial moderada, superior a $25 \%$ e inferior a $75 \%$ e os de dependência fraca, superior a $75 \%$.

Os mapas de isolinhas foram elaborados utilizando-se as informações provenientes dos semivariogramas durante o processo de krigagem na interpolação dos dados. Para a geração dos mapas das variáveis em que não foi verificada dependência espacial foi utilizado o método do inverso do quadrado da distância e não a krigagem.

\section{RESULTADOS E DISCUSSÃO}

Segundo a classificação proposta por Alvarez V. et al. (1999), apenas os valores de pH em água estão bons (Tabela 1). Os níveis dos teores de matéria orgânica, B e Mn estão baixos e os de saturação por bases, $\mathrm{Cu}, \mathrm{Fe}$ e Zn, médios. Essa classificação repetiu-se nas duas profundidades estudadas. Os médios e baixos teores dos micronutrientes sugerem que as práticas de fertilização com esses elementos não foram adequadas e que há alta probabilidade de respostas das culturas à sua aplicação.

Maiores teores de matéria orgânica, Mn e Zn foram encontrados nos primeiros $10 \mathrm{~cm}$ do solo (Tabela 1). Esses resultados são concordantes com os encontrados por Zanão Júnior et al. (2007) para matéria orgânica, Mn e Zn. Esses autores avaliaram a variabilidade espacial dos teores de micronutrientes e matéria orgânica e valores de $\mathrm{pH}$ em um Latossolo Vermelho distrófico de textura muito argilosa, no sistema plantio direto, também por oito anos.

O possível aumento no teor da matéria orgânica nos primeiros $10 \mathrm{~cm}$ de solo ocorreu em decorrência do sistema de cultivo empregado. No sistema plantio direto a quan- 
tidade de resíduos vegetais que retorna ao solo é maior que no sistema convencional. Zanão Júnior et al. (2007) também verificaram maiores teores de matéria orgânica nos primeiros centímetros do solo em um solo muito argiloso, porém em maior expressão. A diferença, apesar de significativa no presente trabalho, foi menor. Tal fato se explica pela textura do solo. Segundo Castro (1989), solos mais arenosos, mesmo no sistema plantio direto, não apresentam aumentos significativos no teor de matéria orgânica como os solos argilosos.

Os valores de pH em água apresentaram-se homogêneos nas profundidades estudadas, como resultado da incorporação do calcário na adoção do sistema plantio direto. Também, segundo Caires et al. (1998), a movimentação de $\mathrm{Ca}\left(\mathrm{HCO}_{3}\right)_{2}$ e $\mathrm{Mg}\left(\mathrm{HCO}_{3}\right)_{2}$ para camadas subsuperficiais contribui para o aumento do $\mathrm{pH}$ nessas camadas.

Os teores de manganês e zinco foram menores na profundidade de 10-20 cm, o que pode ser atribuído aos menores teores de matéria orgânica nessa camada. Esses resultados concordam com os obtidos por Castro et al. (1992), Teixeira et al. (2003) e Zanão Júnior et al. (2007). Os maiores teores de Mn e Zn nos primeiros 10 cm de solo também podem ser explicados pela maior quantidade de matéria orgânica na referida camada, uma vez que ela é uma das principais fontes desses nutrientes. Além disso, quase todo ano o $\mathrm{Zn}$ foi adicionado ao solo juntamente com a adubação de plantio, acumulando-se nessa camada. Segundo Horowitz \& Dantas (1976), pode ocorrer adsorção do Zn em função do teor e da qualidade da argila. Esse elemento também permanece vários anos na superfície do solo e seu movimento lateral é bastante reduzido.

A análise descritiva dos dados tem a função de analisar o comportamento geral dos dados e identificar algum problema que possa exercer qualquer influência na análise geoestatística, apesar de considerar esses dados espacialmente independentes. Os resultados dessa análise indicaram distribuição normal, pois os valores de média e mediana estão próximos em todas as variáveis, mostrando distribuições quase simétricas. Outro indicativo da normalidade são os valores de coeficientes de assimetria e curtose próximos de zero (Tabela 1).

Os valores máximos e mínimos dos teores encontrados nas duas profundidades foram semelhantes (Tabela 1). Analisando as amplitudes dos valores dos atributos avaliados pode-se avaliar sua variabilidade. Correlação entre a amplitude e os respectivos coeficientes de variação (CV) dos atributos foi observada por Souza (1992). A amplitude dos dados representou bem seus respectivos coeficientes de variação no presente trabalho. Os menores coeficientes de variação foram observados para $\mathrm{pH}$ em água e os maiores para boro (Tabela 1), concordando com os dados de Zanão Júnior et al. (2007). O valor máximo do $\mathrm{pH}$ em água encontrado na profundidade de 0-10 cm (menor CV) foi 1,24 vezes maior que o valor mínimo. Para boro, por sua vez, na mesma profundidade (maior $\mathrm{CV}$ ), essa diferença foi 6,0 vezes maior.

Tabela 1. Estatística descritiva para $\mathrm{pH}\left(\mathrm{H}_{2} \mathrm{O}\right)$, teores de M.O. (dag $\left.\mathrm{kg}^{-1}\right), \mathrm{B}, \mathrm{Cu}, \mathrm{Fe}, \mathrm{Mn}, \mathrm{Zn}\left(\mathrm{mg} \mathrm{dm}^{-3}\right)$, e saturação por bases (\%) de um Latossolo Vernelho de textura média no sistema plantio direto nas profundidades de 0-10 e $10-20 \mathrm{~cm}$

\begin{tabular}{|c|c|c|c|c|c|c|c|}
\hline Variável & Média ${ }^{(1)}$ & Mediana & Mínimo & Máximo & CV (\%) & Assimetria & Curtose \\
\hline \multicolumn{8}{|c|}{$0-10 \mathrm{~cm}$} \\
\hline $\mathrm{pH} \mathrm{H}_{2} \mathrm{O}$ & $6,06^{\mathrm{ns}}$ & 6,05 & 5,60 & 6,92 & 4,33 & 0,62 & 0,70 \\
\hline M.O. & $1,67^{*}$ & 1,60 & 1,20 & 2,70 & 10,13 & 0,65 & $-0,18$ \\
\hline V\% & $47,37^{\mathrm{ns}}$ & 48,47 & 30,68 & 62,44 & 14,71 & $-0,14$ & $-0,22$ \\
\hline B & $0,24^{\mathrm{ns}}$ & 0,21 & 0,10 & 0,60 & 44,78 & 0,94 & 0,25 \\
\hline $\mathrm{Cu}$ & $0,96^{\mathrm{ns}}$ & 0,90 & 0,40 & 1,80 & 32,88 & 0,39 & $-0,36$ \\
\hline $\mathrm{Fe}$ & $26,26^{\mathrm{ns}}$ & 25,00 & 11,00 & 40,00 & 24,51 & 0,92 & 0,50 \\
\hline $\mathrm{Mn}$ & $4,43^{*}$ & 4,30 & 1,20 & 7,10 & 32,67 & 0,22 & $-0,67$ \\
\hline $\mathrm{Zn}$ & $1,56^{*}$ & 1,50 & 0,80 & 3,30 & 34,06 & 0,88 & 0,81 \\
\hline $\mathrm{pH} \mathrm{H}_{2} \mathrm{O}$ & 6,06 & 6,04 & 5,60 & 6,65 & 4,57 & 0,36 & $-0,55$ \\
\hline M.O. & 1,51 & 1,40 & 1,10 & 2,30 & 10,11 & 0,73 & $-0,70$ \\
\hline V\% & 44,85 & 45,13 & 20,83 & 65,11 & 19,89 & $-0,24$ & $-0,36$ \\
\hline B & 0,24 & 0,21 & 0,10 & 0,55 & 44,31 & 0,83 & 0,01 \\
\hline $\mathrm{Cu}$ & 0,97 & 0,90 & 0,40 & 2,00 & 40,38 & 0,57 & $-0,35$ \\
\hline $\mathrm{Fe}$ & 23,82 & 23,00 & 15,00 & 37,00 & 20,45 & 0,65 & 0,10 \\
\hline $\mathrm{Mn}$ & 2,89 & 2,90 & 1,00 & 5,10 & 35,35 & 0,19 & $-0,26$ \\
\hline $\mathrm{Zn}$ & 1,30 & 1,20 & 0,40 & 2,00 & 44,47 & 0,56 & $-0,35$ \\
\hline
\end{tabular}

(1) Média de 121 amostras; ${ }^{\text {ns }} \mathrm{e}^{*}$ significam diferença não-significativa e significativa a 5 \% de probabilidade pelo teste t Student, respectivamente, na comparação entre as camadas de solo analisadas. 
Com base no critério de Warrick \& Nielsen (1980), as variáveis pH em água e teores de matéria orgânica apresentaram CV baixo $(\mathrm{CV}<12 \%)$ e as outras CV médio $(12,1 \%<$ $\mathrm{CV}<60 \%$ ). Essas classificações se repetiram nas duas profundidades estudadas. Em vários trabalhos também foram encontrados CV baixo para pH em água e matéria orgânica, necessitando-se de pequeno número de subamostras para estimar, com boa representatividade, esses atributos (Souza, 1992; Santos \& Vasconcellos, 1987; Schlindwein \& Anghinoni, 2000; Zanão Júnior et al., 2007). A distribuição superficial da palhada das culturas é, normalmente, feita de forma homogênea pelas colheitadeiras, contribuindo para baixa a média variabilidade horizontal nos teores de matéria orgânica, como também verificado por Santos \& Vasconcellos (1987) e Souza (1992).

São poucos os dados na literatura sobre variabilidade de micronutrientes em sistema de plantio direto. Silva (2000) também encontrou CVs médios para os micronutrientes catiônicos, com exceção do $\mathrm{Zn}(\mathrm{CV}>70 \%)$, além de Silva \& Silveira (2002), Silva et al. (2003) e Zanão Júnior et al. (2007). Os CV's médios podem ser atribuídos ao efeito residual da adubação e ao modo de aplicação contínua de fertilizantes, mecanicamente e na linha de semeadura, que são mantidos ao longo dos cultivos.

O coeficiente de assimetria é utilizado para caracterizar como e quanto a distribuição de frequências se afasta da simetria. Se o valor encontrado para esse coeficiente for zero, a distribuição é simétrica; se positivo, a distribuição é assimétrica à direita; e se negativo, é assimétrica à esquerda. A maioria das variáveis apresentou coeficientes de assimetria positivos, sendo os maiores valores para boro $(0-10 \mathrm{~cm})$, de 0,94, e o menor também para boro $(10-20 \mathrm{~cm})$ de 0,01 . Coeficientes de assimetria negativos foram encontrados em ambas as profundidades, sendo o maior para saturação por bases $(-0,14)$ e o menor para manganês $(-0,67)$, ambos na profundidade de $0-10 \mathrm{~cm}$.

Apesar de se ter encontrado distribuições assimétricas, verifica-se por alguns indícios como valores médios e medianos semelhantes e coeficientes de assimetria e curtose próximos a zero, para a maioria das variáveis avaliadas, que as medidas de tendência central não são dominadas por valores atípicos na distribuição, segundo Cambardella et al. (1994), indicando que os dados estão adequados para aplicação da geoestatística.

Efetuada por meio dos semivariogramas, a análise geoestatística permitiu detectar que a maioria das variáveis apresentou dependência espacial, excetuando-se $\mathrm{pH}$ em água, boro, zinco e ferro na profundidade de 0-10 cm; e pH em água e saturação por bases na profundidade de 10-20 cm (Tabela 2). Quando a variável estudada é independente espacialmente, o seu $\mathrm{C}_{0}$ (efeito pepita) é igual a $\mathrm{C}_{1}+\mathrm{C}_{0}$ (patamar), conhecido como efeito pepita puro (EPP). O EPP é importante e indica distribuição casual, ou seja, variabilidade não explicada ou variação não detectada, e pode ocorrer devido a erros de medidas, de amostragem ou microvariação não-detectada, considerando ser o espaçamento de amostragem utilizado maior que o necessário para detectar dependência espacial (Cambardella et al., 1994). A existência de dependência espacial e de altos valores de alcance para variáveis como V\% na profundidade de 0-10 cm e nenhuma dependência espacial dessa variável na camada de 10-20 cm exemplifica bem esse fato. A calagem é realizada superficialmente no sistema plantio direto, assim a tendência é que haja maior homogeneidade da saturação por bases na superfície do solo $(0-10 \mathrm{~cm})$, o que permitiu, com o espaçamento de coleta de amostras utilizado, detectar dependência espacial nessa profundidade, não ocorrendo o mesmo na camada de $10-20 \mathrm{~cm}$.

Para todas as variáveis que apresentaram dependência espacial na profundidade de $0-10 \mathrm{~cm}$ ajustou-se o modelo gaussiano para saturação por bases, matéria orgânica e cobre e esférico para manganês; na profundidade de 10-20 cm foi ajustado o modelo gaussiano para matéria orgânica, zinco, manganês e ferro e esférico para boro e cobre.

O alcance é uma medida importante no estudo dos semivariogramas para planejamento e avaliação experimental, podendo auxiliar na definição de procedimentos amostrais (Webster, 1985). Ele significa a distância máxima que uma variável se correlaciona espacialmente (Davis, 1986), o que quer dizer que determinações realizadas a distâncias maiores que o alcance apresentam distribuição aleatória e, por isso, são independentes entre si. Os valores de alcance variaram conforme o atributo avaliado. Foram maiores na profundidade de $0-10 \mathrm{~cm}$ e menores na de $10-20 \mathrm{~cm}$. O menor valor de alcance foi encontrado para boro, sendo $300 \mathrm{~m}(10-20 \mathrm{~cm})$, e o maior para matéria orgânica, $539 \mathrm{~m}(0-10 \mathrm{~cm})$. Se for adotada a geoestatística no esquema de amostragem, o número de amostras a serem coletadas em cada profundidade será distinto, de acordo com a profundidade e o atributo a ser avaliado. Portanto, além da variabilidade vertical, há também variabilidade horizontal entre as profundidades.

Para analisar o grau de dependência espacial dos atributos em estudo, utilizou-se a classificação proposta por Cambardella et al. (1994). A análise do efeito pepita dos atributos do solo revelou que ocorreu apenas dependência espacial moderada nas duas profundidades. Tal fato 
demonstra que os semivariogramas explicam a maior parte da variância dos dados.

Os mapas de isolinhas obtidos por meio da interpolação dos dados, pelo método da krigagem, são fundamentais na agricultura de precisão, pois são posteriormente analisados e trabalhados com a finalidade de se planejar amostragem e realizar adubações mais exatas, com menor relação custo/benefício, de acordo com a variabilidade espacial dos valores de cada atributo químico e físico do solo. Nesses mapas, pode-se visualizar na área amostrada a distribuição espacial dos teores dos elementos avaliados. O conhecimento dos valores do alcance e a visualização das localizações das áreas nos mapas de isolinhas são importantes para o planejamento do manejo da fertilidade do solo.

Foram construídos mapas de isolinhas de acordo com as classes de fertilidade do solo, conforme classificação proposta por Alvarez V. et al., (1999) para os solos de Minas Gerais (Figuras 1 e 2). Fazer os mapas de acordo com as classes de fertilidade do solo propostas para cada estado ou região é mais lógico do que fazê-los de acordo com os intervalos propostos pelos programas que os geram, ou intervalos delimitados arbitrariamente. Isso porque as recomendações de adubação atuais são realizadas de acordo com as classificações dos níveis dos nutrientes no solo. Sugere-se que todos os mapas gerados em trabalhos que estudam a variabilidade espacial dos atributos químicos dos solos sejam então criados de acordo com os critérios ou classes que serão considerados no momento da recomendação da adubação.

No caso do $\mathrm{pH}$ em água, nas duas profundidades pode-se observar que ele foi classificado como "bom" em toda a área amostrada. Assim, para o pH em água existe tanto homogeneidade vertical quanto horizontal. Os teores de matéria orgânica de 0-10 cm e saturação por bases, nas duas profundidades, também estão quase homogêneos, existindo pequenas áreas de outra classe. A maioria da área está com teores de matéria orgânica e saturação por bases considerados baixos e médios, respectivamente. Na profundidade de $10-20 \mathrm{~cm}$, em toda a área os teores de matéria orgânica foram classificados como baixos (Figura 1).

No caso do boro, existem três classes contempladas no mapa nas duas profundidades. A predominante demonstra que os teores desse micronutriente estão baixos, existindo pequenas manchas das classes “muito baixo” e “médio" (Figura 1). Cobre, manganês (0-10 cm) e zinco são elementos que possuem os mapas de isolinhas mais heterogêneos, com áreas muito proporcionais de três classes de interpretação dos níveis dos teores desses micronutrientes: "muito baixo”, "baixo” e "médio” (Figura 2). Os mapas de isolinhas dos teores de Fe evidenciaram que existem apenas duas classes: “médio” e "bom”, predominando a primeira. Aliás, na profundidade de 10-20 cm existe apenas uma minúscula área em que os teores de Fe estão bons (Figura 2).

Tabela 2. Estmativas dos parâmetros dos modelos dos semivariogramas ajustados para as variáveis $\mathrm{pH}\left(\mathrm{H}_{2} \mathrm{O}\right)$, M.O. (dag kg-1), B, $\mathrm{Cu}, \mathrm{Fe}, \mathrm{Mn}$ e $\mathrm{Zn}\left(\mathrm{mg} \mathrm{dm}^{-3}\right)$ nas profundidades de 0-10, 10-20 e 0-20 cm

\begin{tabular}{|c|c|c|c|c|c|c|}
\hline Variável & $\mathrm{C}_{0}{ }^{(1)}$ & $\mathrm{C}_{1}+\mathrm{C}_{0}^{(2)}$ & $\begin{array}{c}\text { Alcance } \\
\text { (m) }\end{array}$ & Modelo & $\begin{array}{c}\text { Dependência } \\
{\left[\mathrm{C}_{0} / \mathrm{C}_{0}+\mathrm{C}_{1}\right] \times 100}\end{array}$ & Classificação \\
\hline \multicolumn{7}{|c|}{$0-10 \mathrm{~cm}$} \\
\hline $\mathrm{pH} \mathrm{H}_{2} \mathrm{O}$ & 0,0717 & 0,0717 & - & $\mathrm{Epp}^{(3)}$. & - & - \\
\hline M.O. & 0,0508 & 0,1515 & 539 & $\mathrm{Gau}^{(4)}$ & 33,53 & $\mathbf{M}^{(6)}$ \\
\hline V\% & 33,6805 & 50,9582 & 500 & Gau. & 66,06 & M \\
\hline B & 0,0110 & 0,0110 & - & Epp. & - & - \\
\hline $\mathrm{Cu}$ & 0,6123 & 0,9812 & 516 & Gau. & 62,40 & M \\
\hline $\mathrm{Fe}$ & 43,1232 & 43,1232 & - & Epp. & - & - \\
\hline $\mathrm{Mn}$ & 0,6032 & 2,1721 & 500 & $\operatorname{Esf}^{(5)}$ & 27,77 & M \\
\hline $\mathrm{Zn}$ & 0,2703 & 0,2703 & - & Epp. & - & - \\
\hline $\mathrm{pH} \quad \mathrm{H}_{2} \mathrm{O}$ & 0,0789 & 0,0789 & - & Epp. & - & - \\
\hline M.O. & 0,0621 & 0,1244 & 496 & Gau. & 49,92 & M \\
\hline V\% & 66,6745 & 66,6745 & - & Epp. & - & - \\
\hline B & 0,0075 & 0,0127 & 300 & Esf. & 59,06 & M \\
\hline $\mathrm{Cu}$ & 0,0902 & 0,1562 & 314 & Esf. & 57,75 & M \\
\hline $\mathrm{Fe}$ & 15,0222 & 25,0331 & 310 & Gau. & 60,01 & M \\
\hline $\mathrm{Mn}$ & 0,7042 & 0,7042 & - & Epp. & - & - \\
\hline
\end{tabular}


$0-10 \mathrm{~cm}$

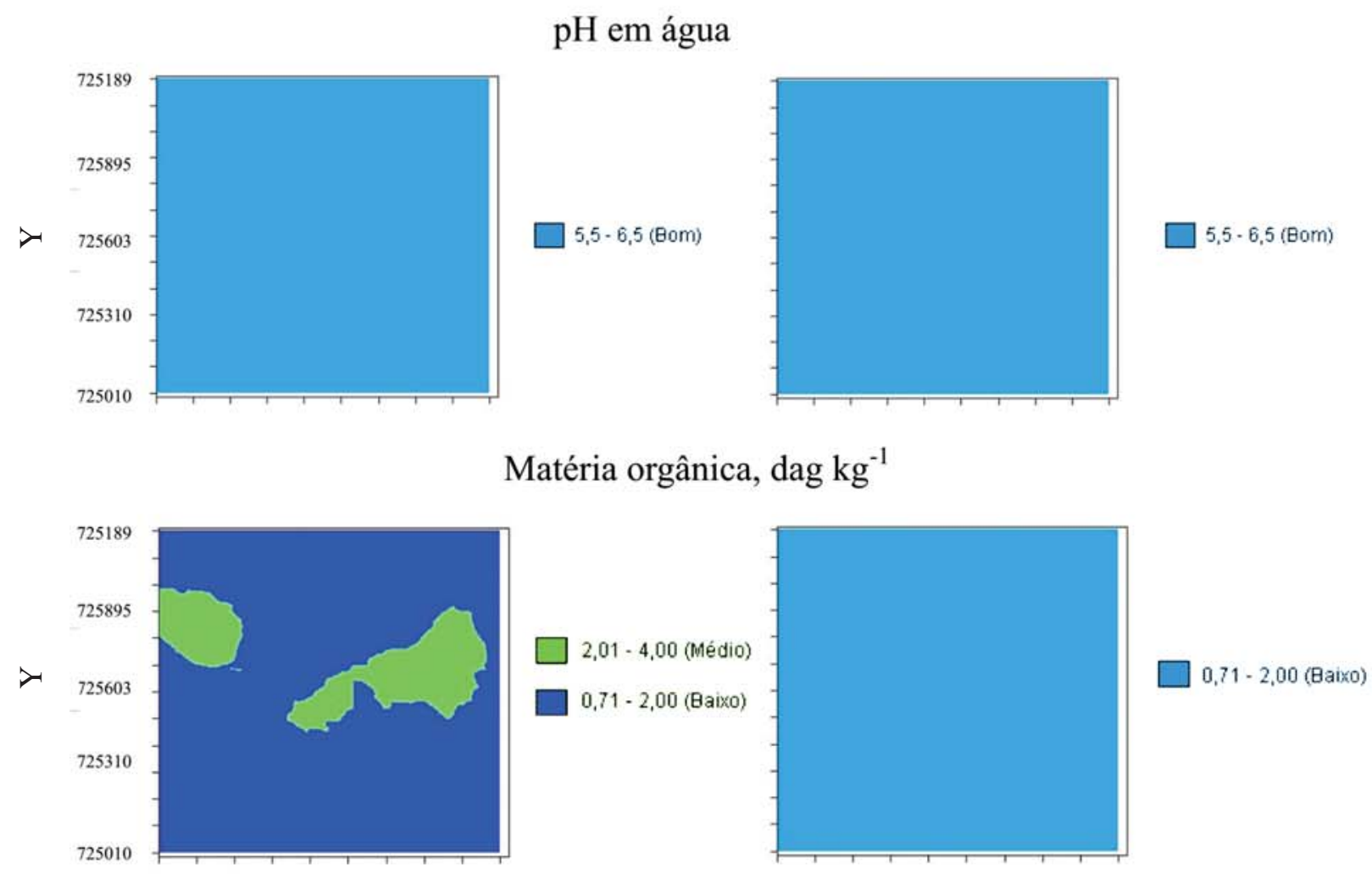

Saturação por bases, $\%$
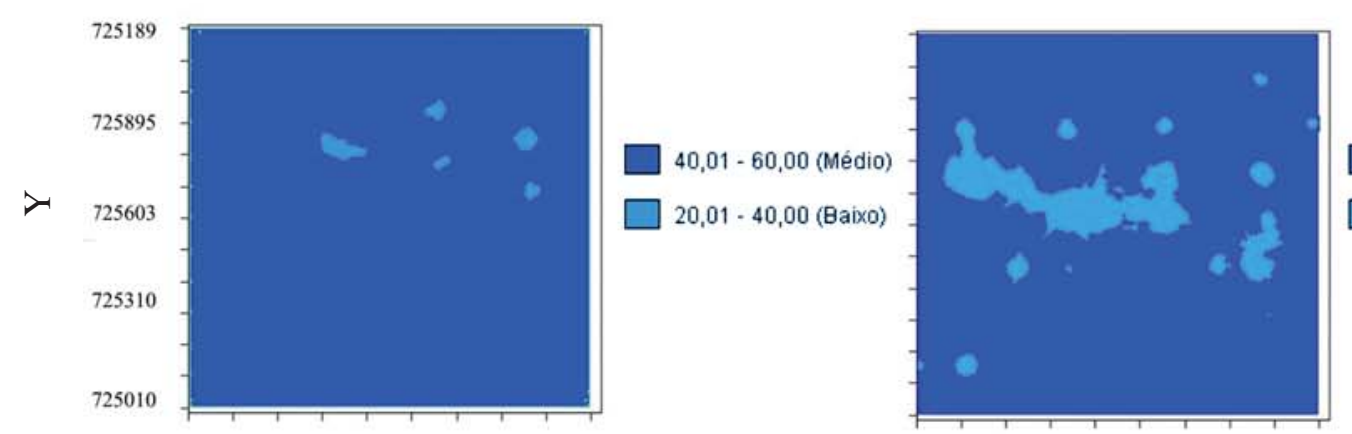

$40,01-60,00$ (Médio)

$20,01 \cdot 40,00$ (Baixo)

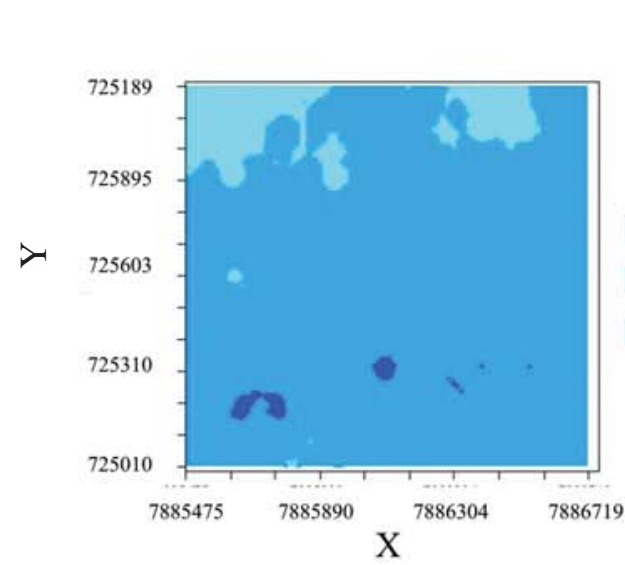

\section{Boro, $\mathrm{mg} \mathrm{dm}^{-3}$}

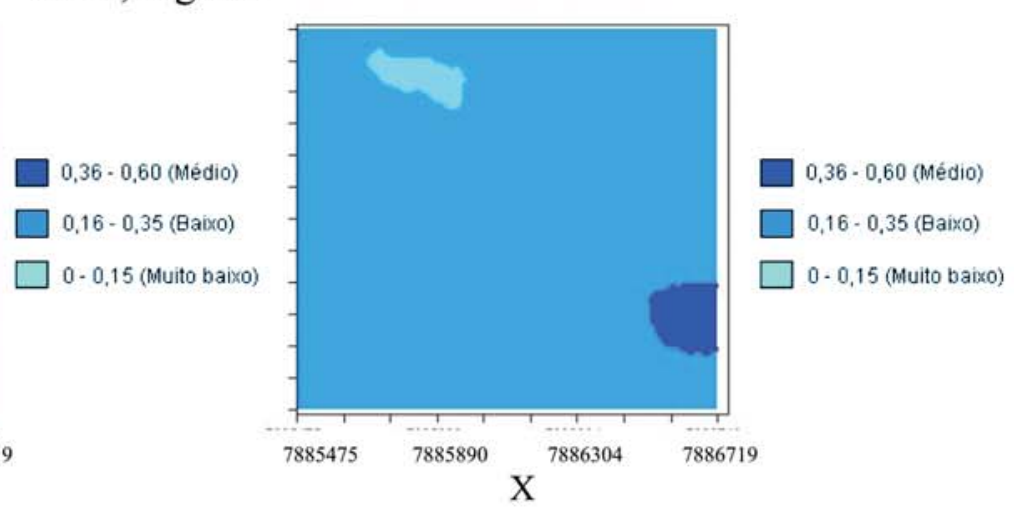

Figura 1. Mapas de isolinhas baseados nas classes de valores de pH em água, teores de matéria orgânica, saturação por bases e teores de boro (segundo Alvarez V. et al., 1999), na profundidade de 0-10 e 10-20 cm, em Latossolo Vemelho, textura média, no sistema plantio direto. 


$$
0-10 \mathrm{~cm}
$$

\section{Cobre, $\mathrm{mg} \mathrm{dm}^{-3}$}
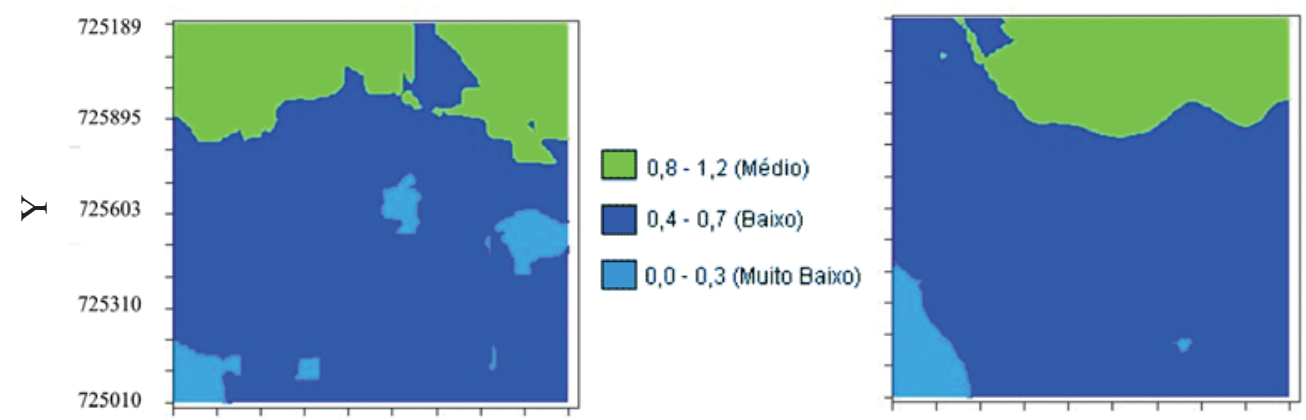

Ferro, $\mathrm{mg} \mathrm{dm}^{-3}$
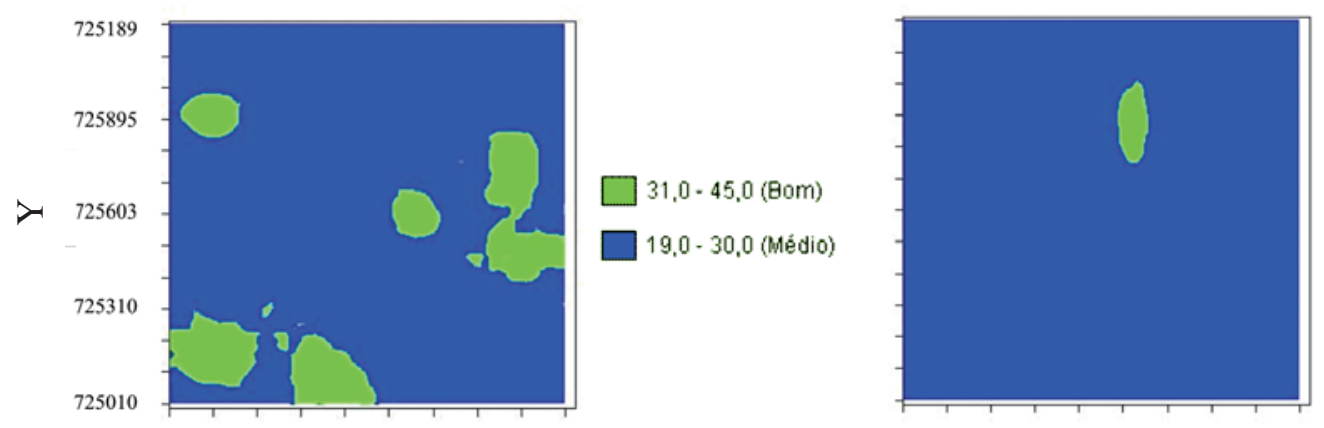

0,8-1,2 (Médio)

$0,4 \cdot 0,7$ (Baixo)

$0,0 \cdot 0,3$ (Muito Baixo)

$31,0 \cdot 45,0(80 m)$

$19,0 \cdot 30,0$ (Médio)

Manganês, $\mathrm{mg} \mathrm{dm}^{-3}$
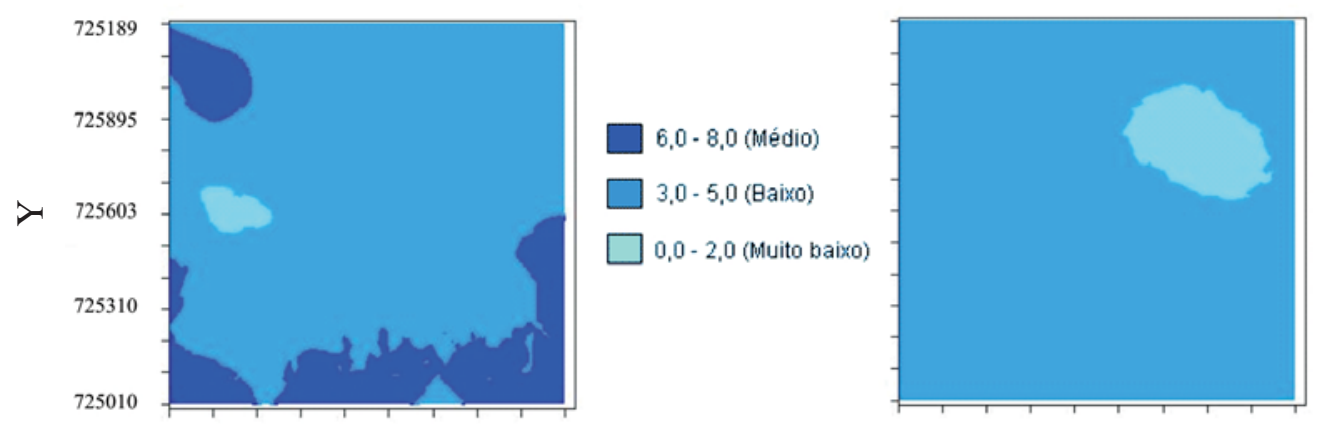

$3,0 \cdot 5,0($ (Baixo)

$0,0-2,0$ (Muito baixo)

Zinco, $\mathrm{mg} \mathrm{dm}^{-3}$
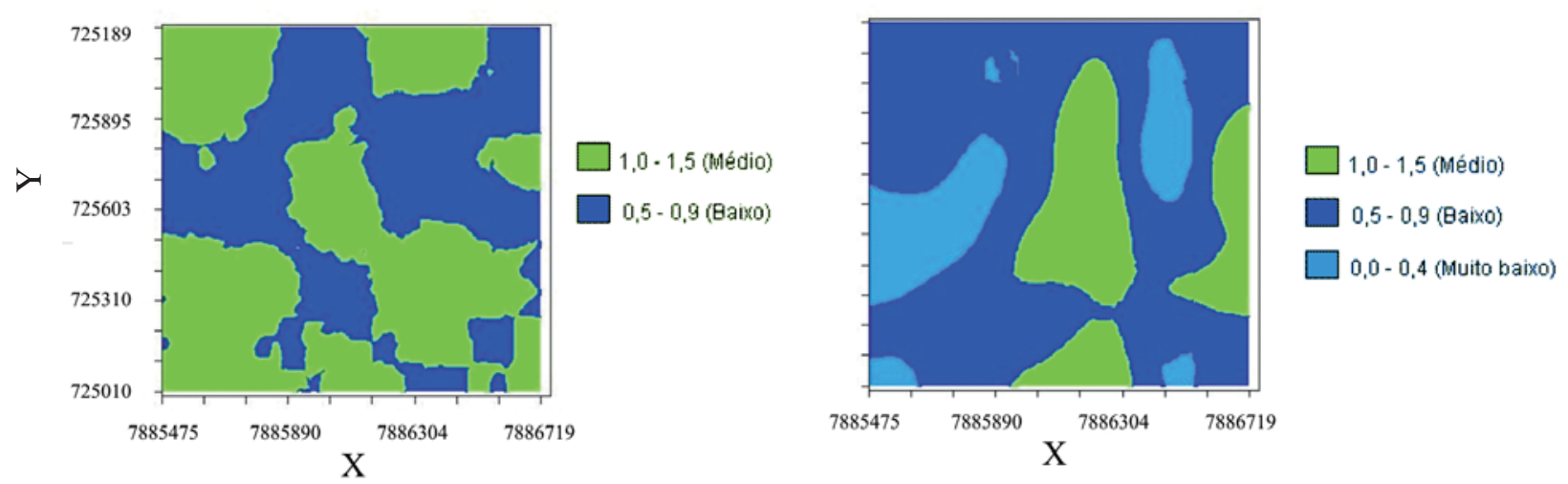

Figura 2. Mapas de isolinhas baseados nas classes de teores de cobre, ferro, manganês e zinco (segundo Alvarez V. et al., 1999) na profundidade de 0-10 e 10-20 cm, em Latossolo Vemelho, textura média, no sistema plantio direto. 
Assim, com o auxílio desses mapas, e com a espacialização das classes de interpretação dos teores dos atributos químicos do solo possibilita-se elaborar estratégias de manejo de calagem e adubação de modo que se eleve a fertilidade e se tenha no futuro apenas a classe mais adequada para a cultura a ser implantada, como no caso do pH em água. A partir desse momento a área pode ser considerada homogênea e menos amostras de solo coletadas para representar a sua fertilidade.

\section{CONCLUSÕES}

A dependência espacial varia conforme o atributo químico avaliado e a profundidade de coleta.

Além da variabilidade vertical, há também variabilidade horizontal entre as profundidades, visto que para um mesmo nutriente o alcance foi diferente entre as camadas amostradas.

Os valores de alcance foram maiores na profundidade de $0-10 \mathrm{~cm}$.

\section{AGRADECIMENTOS}

À FAPEMIG, pela bolsa de iniciação científica e financiamento concedidos e, aos técnicos do Laboratório de Análise de Solos do Instituto de Ciências Agrárias da Universidade Federal de Uberlândia, pelo auxílio na realização das análises químicas.

\section{REFERÊNCIAS}

Alvarez V VH, Novais RF, Barros NF, Cantarutti RB \& Lopes AS (1999) Interpretação dos resultados das análises de solos. In: Ribeiro AC, Guimarães PTG \& Alvarez V VH (Eds). Recomendações para o uso de corretivos e fertilizantes em Minas Gerais, $5^{\text {a }}$ Aproximação. Viçosa, Comissão de Fertilidade do Solo do Estado de Minas Gerais, p.25-32.

Bekele A \& Hudnall WH (2006) Spatial variability of soil chemical properties of a prairie-forest transition in Louisiana, Plant and Soil, 280:7-21.

Bhatti AU, Mulla DJ \& Frazier BE (1991) Estimation of soil properties and wheat yields on complex eroded hills using geoestatistics and thematic mapper images. Remote Sensing Environment, 37:181-191.

Burgess TM \& Webster R (1980) Optimal interpolation and isarithmic mapping of soil properties. In: The semivariogram and punctual kriging. Journal Soil Science, 31:315-331.

Burrough PA, Varekamp C \& Skidmore, AK (1996) Using public domain geostatistical and GIS software for spatial interpolation. Photogrammetric. Engineering \& Remote Sensing, 62:845-854.

Caires EF, Chueiri WA, Madruga EF \& Figueiredo A (1998) Alterações de características químicas do solo e resposta da soja ao calcário e gesso aplicados na superfície em sistema de cultivo sem preparo de solo. Revista Brasileira de Ciência do Solo, 22:27-34
Cambardella CA, Moorman TB, Novak JM, Parkin TB, Karlen DL, Turco RF \& Konopka AE (1994) Field scale variability of soil properties in Central Iowa soils. Soil Science Society of American Journal, 58:1501-1511.

Campos MCC, Marques Júnior J, Pereira GT, Souza ZM \& Barbieri DM (2008) Aplicação de adubo e corretivo após o corte da cana-planta utilizando técnicas Geoestatísticas, Ciência Rural, 38:974-980

Castro OM (1989) Sistemas de preparo do solo para a cultura do milho. Campinas, Fundação Cargill. 41 p. (Série técnica, 3).

Castro OM, Camargo OAC, Cantarella H, Vieira SR \& Dechen SC (1992) Teores de zinco, cobre, manganês e ferro em dois Latossolos sob plantio direto e convencional. Bragantia, 51:77-84.

Cavalcante EGS, Alves MC, Pereira GT \& Souza ZMS (2007) Variabilidade espacial de MO, P, K e CTC do solo sob diferentes usos e manejos, Ciência Rural, 37:394-400.

Corá JE, Araújo AV, Pereira GT \& Beraldo JMG (2004) Variabilidade espacial de atributos do solo para adoção do sistema de agricultura de precisão na cultura de cana-de-açúcar. Revista Brasileira de Ciência do Solo, 28:1013-1021.

Davis JC (1986) Statistics and data analysis in geology. 2.ed., New York, Jonh Wiley. 7646 p.

Gamma Design Software. Geoestatistics for the environmental sciences (version 5.1 for windows). Michigan: 2000. CD ROM.

Horowitz A \& Dantas HS (1976) Geoquímica dos elementos menores nos solos de Pernambuco. IV. Zinco na zona LitoralMata. Pesquisa Agropecuária Brasileira 11:27-35.

Motomiya AVA, Corá JE \& Pereira GT (2006) Uso da krigagem indicatriz na avaliação de Indicadores de fertilidade do solo, Revista Brasileira de Ciência do Solo, 30:485-496.

Reichardt K, Vieira SR \& Libardi PL (1986) Variabilidade espacial de solos e experimentação de campo, Revista Brasileira de Ciência do Solo, 10:1-6.

Salet RL, Kray CH, Fornari TG, Conte E, Kochhann RA \& Anghinoni I (1996) Variabilidade horizontal e amostragem de solo no sistema plantio direto. In: Reunião Sul-Brasileira de Ciência do Solo, Lages. Resumos Expandidos, Sociedade Brasileira de Ciência do Solo. p.74-76.

Santos HL \& Vasconcellos CA (1987) Determinação do número de amostras de solo para análise química em diferentes condições de manejo. Revista Brasileira de Ciência do Solo, 11:97-100.

Schlindwein JÁ \& Anghinoni I (2000) Variabilidade horizontal de atributos de fertilidade e amostragem do solo no sistema plantio direto. Revista Brasileira de Ciência do Solo, 24.85-91.

Silva CC \& Silveira PM (2002) Influência de sistemas agrícolas em características químico-físicas do solo. Ciência e Agrotecnologia, 26:505-515.

Silva FC (1999) Manual de análises químicas de solos, plantas e fertilizantes. Brasília, EMBRAPA. 370 p.

Silva CA (2000) Amostragem e variabilidade de atributos de solo e planta em área de soja sob plantio direto. In: Fertibio 2000, Santa Maria. Resumos Expandidos, Sociedade Brasileira de Ciência do Solo. CD-ROM.

Silva MAG, Muniz AS, Sengik E, Mata JDV, Carissimi C \& Cegana AC (2003) Amostragem e variabilidade nos atributos de fertilidade em um latossolo sob plantio direto em São Miguel do Iguaçu, Estado do Paraná. Acta Scientiarum: Agronomy, 25: 243-248.

Souza LS (1992) Variabilidade espacial do solo em sistemas de manejo. Tese de Doutorado. Universidade Federal do Rio Grande do Sul, Porto Alegre, 162 p. 
Souza LS, Cogo NP \& Vieira SR (1997) Variabilidade de propriedades físicas e químicas do solo em um pomar cítrico. Revista Brasileira de Ciência do Solo, 21:367-372.

Souza ZM, Marques Júnior J, Pereira GT \& Montanari R (2006) Otimização amostral de atributos de latossolos considerando aspectos solo-relevo, Ciência Rural, 36:829-836.

Teixeira IR, Souza CM, Borém A \& Silva GF (2003) Variação dos valores de $\mathrm{pH}$ e dos teores de carbono orgânico, cobre, manganês, zinco e ferro em profundidade em Argissolo Vermelho-Amarelo, sob diferentes sistemas de preparo de solo. Bragantia, 62:119-126.

Trevisan LR, Lana RMQ, Zanão Júnior LA, Lacerda ZC \& Guimarães EC (2008) Variabilidade vertical de $\mathrm{pH}$, bases e enxofre em Latossolo Vermelho cultivado sob sistema plantio direto e cultivo mínimo. Horizonte Científico, 7:1-11.
Vieira SR, Hatfield JL, Nielsen, DR \& Biggar JW (1983) Geostatitical theory and application to variability of some agronomical properties. Hilgardia, 51:1-75.

Warrick AW \& Nielsen DR (1980) Spatial variability of soil physical properties in the field. In: Hillel D. (Ed.) Applications of soil physics. New York, Academic Press. p.319-44.

Webster R (1985) Quantitative spatial analysis of soil in the field. In: Stewart BA (Ed.). Advance in soil science. New York, Springer-Verlag, p.1-70.

Zanão Júnior LA, Lana RMQ \& Guimarães EC (2007) Variabilidade espacial do $\mathrm{pH}$, teores de matéria orgânica e micronutrientes em profundidade em um Latossolo Vermelho sob semeadura direta. Ciência Rural, 37:10001007 . 